The setting is a rural district hospital in Yala province, the southernmost province of Thailand that shares an international border with Malaysia, another country where COVID-19 outbreaks occur. This small, 30-bed hospital serves local people in that rural district. The outbreak occurred on March 22, 2020, when 3 medical personnel ( 2 nurses and 1 physician) developed fever and COVID-19 was confirmed after they had provided regular care to local people with a history of COVID-19 contact. All 3 medical personnel are presently under respiratory isolation, but none has had lung complications. All of the other 21 medical personnel of the hospital, including 7 physicians, are under quarantine. The hospital had to be closed and no longer provides any service. According to our best knowledge, this is the first report of COVID-19 outbreak in a hospital that resulted in total disruption of hospital function.

The COVID-19 outbreak in hospitals is serious because it can result in abrupt cessation of local medical care and especially management of COVID-19 during the outbreak. Hospitals usually have protective systems in place, but a high-volume load might result in unsuccessful disease control. A good hospital infection control program during COVID-19 should be simple but strict, with aggressive procedures that might differ from routine clinical practice. Strategic planning to reduce unnecessary physical examinations, to implement universal drug distribution, or to postpone unnecessary procedures during the crisis period, is necessary.
Acknowledgments.

Financial support. No financial support was provided relevant to this article.

Conflicts of interest. All authors report no conflicts of interest relevant to this article.

\section{References}

1. Hsia, W. Emerging new coronavirus infection in Wuhan, China: situation in early 2020. Case Study Case Rep 2020;10:8-9.

2. Huh S. How to train the health personnel for protecting themselves from novel coronavirus (COVID-19) infection during their patient or suspected case care. J Educ Eval Health Prof 2020 [Epub 2020 Mar 7]. doi: 10.3352/ jeehp.2020.17.10.

3. Glauser W. Proposed protocol to keep COVID-19 out of hospitals. CMAJ 2020;192:E264-E265.

4. Joob B, Wiwanitkit V. COVID-19 in medical personnel: observation from Thailand. J Hosp Infect 2020;104:453.

5. Liu M, He P, Liu HG, et al. Clinical characteristics of 30 medical workers infected with new coronavirus pneumonia [in Chinese]. Zhonghua Jie He He Hu Xi Za Zhi. 2020;43:209-214.

6. Yasri S, Wiwanitkit V. Editorial: Wuhan coronavirus outbreak and imported case. Adv Trop Med Pub Health Int 2020;10(1):1-2.

\title{
Electronic screening through community engagement: A national strategic plan to find COVID-19 patients and reduce clinical intervention delays
}

\author{
Mehrdad Amir-Behghadami MSc ${ }^{1,2,3}$ and Masoumeh Gholizadeh $\mathrm{PhD}^{1,2}$ \\ ${ }^{1}$ Tabriz Health Services Management Research Center, Health Management and Safety Promotion Research Institute, Tabriz University of Medical Sciences, \\ Tabriz, Iran, ${ }^{2}$ Iranian Center of Excellence in Health Management (ICEHM), Department of Health Service Management, School of Management and Medical \\ Informatics, Tabriz University of Medical Sciences, Tabriz, Iran and ${ }^{3}$ Student Research Committee (SRC), Tabriz University of Medical Sciences, Tabriz, Iran
}

To the Editor-Coronavirus disease 2019 (COVID-19) began circulating in Wuhan, Hubei Province, China, in December 2019. Evidence of human-to-human transmission has been reported in both communities and hospitals ${ }^{1}$; COVID-19 is a highly contagious disease that can spread rapidly through respiratory droplets of infected individuals. ${ }^{2}$ According to one of the first published COVID-19 studies, the most common symptoms at onset are fever, cough, myalgia, and/or fatigue, and less common symptoms include sputum, headache, hemoptysis, and diarrhea. ${ }^{3}$ However, these symptoms may be more severe in the elderly, the immunosuppressed, and those with chronic diseases including diabetes, cardiovascular diseases, cancer, and pulmonary dysfunction. ${ }^{4,5}$

Author for correspondence: Dr Masoumeh Gholizadeh, Iranian Center of Excellence in Health Management, School of Management and Medical Informatics, Tabriz University of Medical Sciences, University Rd, Golbad, EAZN 5165665811, Tabriz, East Azerbaijan, Iran, E-mail: Gholizadehm@tbzmed.ac.ir.

Cite this article: Amir-Behghadami M and Gholizadeh M. (2020). Electronic screening through community engagement: A national strategic plan to find COVID-19 patients and reduce clinical intervention delays. Infection Control \& Hospital Epidemiology, 41: 1476-1478, https://doi.org/10.1017/ice.2020.188
The global pandemic is evolving dynamically. On January 30, 2020, the World Health Organization (WHO) declared that COVID-19 is a "Public Health Emergency of International Concern (PHEIC)" during its second meeting of the Emergency Committee. ${ }^{6}$ As of February 27, 2020, there were 1,610,909 accumulative confirmed cases and 99,690 cumulative deaths globally.? Iranian health authorities confirmed the first COVID-19 cases on February 19, 2020, in Qom. As of February 27, 2020, there had been $>66,220$ confirmed cases of COVID-19 and 4,110 deaths in Iran. ${ }^{8}$ Notably, however, Iran has had the highest improvement in COVID-19; it ranks second in the world after China. Recently, although the number of new cases reported in China has been steadily decreasing, epidemics in other countries are still a major concern. Prevention and identification of the disease have become the most important tasks in Iran, and the government has invested many material and human resources to manage the epidemic. ${ }^{9}$ Currently, no licensed preventative vaccine or specific antiviral therapy is available for COVID-19, and according to the basic theory of controlling infectious diseases, the most effective measures include eliminating the source of infection, disrupting

(c) 2020 by The Society for Healthcare Epidemiology of America. All rights reserved This is an Open Access article, distributed under the terms of the Creative Commons Attribution licence (http://creativecommons.org/licenses/by/4.0/), which permits unrestricted re-use, distribution, and reproduction in any medium, provided the original work is properly cited. 
Table 1. Indicators of Screened, Infected, and Death Cases

\begin{tabular}{|c|c|}
\hline Screening (March 4 through April 7, 2020) & Value \\
\hline No. of services delivered for COVID-19 & $70,141,824$ \\
\hline Target population with symptoms, $\%$ & 1.45 \\
\hline Screened symptomatic cases referred to clinic, \% & 0.2 \\
\hline Visited cases that required homecare, \% & 23.9 \\
\hline Visited cases that received dual medication, $\%$ & 0.6 \\
\hline Visited cases that referred to the hospital, \% & 4.5 \\
\hline People visiting hospital and are admitted in the hospital, \% & 29.2 \\
\hline Client satisfaction with the received services, \% & 97.3 \\
\hline \multicolumn{2}{|l|}{ New lab-confirmed cases (in the previous $72 \mathrm{~h}$ ) } \\
\hline Age, mean y (standard deviation) & $54.8(18.5)$ \\
\hline Age, median y (interquartile range) & $55(39-68)$ \\
\hline \multicolumn{2}{|l|}{ Sex distribution, \% } \\
\hline Male & 49.6 \\
\hline Female & 50.4 \\
\hline Cases with at least 1 comorbidity, $\%$ & 26.1 \\
\hline Cases admitted in ICU, $\%^{a}$ & 11.6 \\
\hline Cases with more severe forms of the disease, $\%^{b}$ & 15.4 \\
\hline \multicolumn{2}{|l|}{ COVID-19 deaths (in the previous week) ${ }^{c}$} \\
\hline Age, mean y (standard deviation) & $69.6(15.1)$ \\
\hline Age, median y (interquartile range) & $71(61-81)$ \\
\hline Cases aged $>60 \mathrm{y}, \%$ & 77.3 \\
\hline \multicolumn{2}{|l|}{ Sex distribution (\%) } \\
\hline Male & 58.4 \\
\hline Female & 41.6 \\
\hline Cases with at least 1 comorbidity, $\%$ & 44.2 \\
\hline Cases aged $>60$ y with at least 1 comorbidity, $\%$ & 87.7 \\
\hline
\end{tabular}

Note. COVID-19, coronavirus 2019; ICU, intensive care unit.

${ }^{\text {a }}$ o the total number of hospitalized COVID-19 patients.

b Based on available data, we considered patients with death outcome, as well as those admitted to ICU or under mechanical ventilation, as more severe cases. The information in this chart is based on hospitalized cases, and outpatients are not included in this calculation. Inclusion of outpatients and asymptomatic cases would decrease the proportion of severe cases.

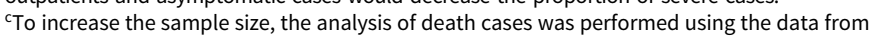
the previous week. Reference: Daily Situation Report on COVID-19, Ministry of Health and Medical Education, IR Iran.

transmission, and protecting susceptible individuals. Therefore, to cope with a sudden outbreak of COVID-19, the community needs to be screened, and whether the infection has occurred and the dynamics of when it is contagious need to be understood more fully.

The Iranian Ministry of Health and Medical Education designed and has been implementing an electronic national screening system (https://salamat.gov.ir/) using a modern information network technology. ${ }^{10}$ After logging information (eg, national code, date of birth, phone number) into the system, Iranian residents answer some questions about COVID-19 symptoms, immunosuppression, and some chronic diseases, as well as the presence of others suspected of having COVID-19 disease among their relatives. Those suspected of having the disease receive a message regarding their health status, and healthcare providers then call them and guide them. Also, the their residences are disinfected and other family members are quarantined if required. If they do not improve within 3 days, they are referred to the emergency department of a hospital. Some screening-related information is provided in Table 1.

This self-screening plan has been successful through government implementation and community engagement. During the pandemic, many efforts have been made to find effective and efficient solutions for the initial management of COVID-19 globally. One of the significant factors, which is been emphasized today, is the important role of community engagement in the management and screening of infected patients. People's attitudes toward the disease and understanding of its consequences if left untreated have played an important role in encouraging their participation in self-screening through designed website. The government has allocated $>17,000$ health houses and $>9,000$ comprehensive health centers in urban, suburban, and rural areas throughout the country to support the plan. These centers, as community health centers, play an important role in these efforts; they are responsible for delivering integrated care services to the population in geographically defined areas. ${ }^{11}$ In addition, this plan is consistent with the overall goal of developing health systems, strengthening their capacity to meet the needs of the community, and achieving universal health coverage.

In conclusion, a successful electronic screening system was developed and introduced to combat the COVID-19 pandemic in Iran. On one hand, this system helps in the initial identification of patients with COVID-19 infections and prevents any delay in clinical interventions. On the other hand, it prevents nonemergency referrals to emergency departments of hospitals. Implementing a simple strategy can be effective for the health system in dealing with this pandemic. Therefore, sharing our successful experience, which was the result of good cooperation and cohesion between the government and community, may be helpful for authorities in other countries.

\section{Acknowledgments.}

Financial support. No financial support was provided relevant to this article.

Conflicts of interest. All authors report no conflicts of interest relevant to this article.

\section{References}

1. Nishiura H, Jung S-m, Linton NM, et al. The extent of transmission of novel coronavirus in Wuhan, China, 2020. J Clin Med 2020;9:330.

2. Huang C, Wang Y, Li X, et al. Clinical features of patients infected with 2019 novel coronavirus in Wuhan, China. Lancet 2020;395:497-506.

3. Chen N, Zhou M, Dong X, et al. Epidemiological and clinical characteristics of 99 cases of 2019 novel coronavirus pneumonia in Wuhan, China: a descriptive study. Lancet 2020;395:507-513.

4. Wang X, Zhou Z, Zhang J, et al. A case of 2019 novel coronavirus in a pregnant woman with preterm delivery. 2020. Clin Infect Dis $2020 \mathrm{Feb}$ 28 [Epub ahead of print]. doi: 10.1093/cid/ciaa200.

5. Amir-Behghadami M, Janati A. The importance of designing and implementing a participatory surveillance system: an approach to early detection and prevention of novel coronavirus (2019-nCov). Am J Infect Control 2020 Apr 1 [Epub ahead of print]. doi: 10.1016/j.ajic.2020.03.013.

6. Novel coronavirus (2019-nCoV) situation report - 38. World Health Organization website. https://www.who.int/docs/default-source/coronaviruse/situationreports/20200227-sitrep-38-covid-19.pdf?sfvrsn=9f98940c_2. Published February 27, 2020. Accessed March 28, 2020.

7. Coronavirus disease 2019 (COVID-19) situation report - 82. World Health Organization website. https://www.who.int/docs/default-source/ 
coronaviruse/situation-reports/20200411-sitrep-82-covid-19.pdf?sfvrsn= 74a5d15_2. Published April 11, 2020. Accessed April 12, 2020.

8. COVID-19 daily epidemiology journal. Iranian Ministry of Health and Medical Education website. http://corona.behdasht.gov.ir/files/site1/files/ Factsheet-20-1399.01.21-En.pdf. Accessed April 12, 2020.

9. Arab-Mazar Z, Sah R, Rabaan AA, Dhama K, Rodriguez-Morales AJ. Mapping the incidence of the COVID-19 hotspot in Iran-implications for travellers. Trav Med Infect Dis 2020 [Epub ahead of print]. doi: 10. 1016/j.tmaid.2020.101630.
10. Home-to-home program to be launched to combat COVID-19. Tehran Times website. https://www.tehrantimes.com/news/445750/Home-tohome-program-to-be-launched-to-combat-COVID-19. Published March 2, 2020. Accessed April 30, 2020.

11. Tabrizi JS, Farahbakhsh M, Sadeghi-Bazargani H, Hassanzadeh R, Zakeri A, Abedi L. Effectiveness of the health complex model in Iranian primary health care reform: the study protocol. Patient Prefer Adherence 2016; 10:2063.

\title{
Action and problems related to the COVID-19 outbreak in India
}

\author{
Pooja Sharma MTech ${ }^{1}$ and Karan Veer $\mathrm{PhD}^{1}$ (1) \\ ${ }^{1}$ Department of Instrumentation \& Control Engineering, Dr B. R. Ambedkar National Institute of Technology, Jalandhar, Punjab, India
}

To the Editor - Named SARS-CoV-2 by the International Committee on Taxonomy of Viruses, this novel coronavirus attacks the lower respiratory tract of patients infected with cryptogenic organizing pneumonia. ${ }^{1}$ The infectious disease it causes was named COVID-19 by the World Health Organization. Coronaviruses comprise a large family of viruses that cause illness ranging from the common cold to more severe diseases like pneumonia, Middle East respiratory syndrome (MERS), and severe acute respiratory syndrome (SARS). Most people are vulnerable to SARS-CoV-2, and this novel coronavirus can affect people with low or normal immunity. ${ }^{2}$ People with low immunity, such as the elderly, pregnant women, and patients with chronic diseases, are prone to severe acute symptoms after contracting COVID-19. SARS-CoV-2 is mainly transmitted via droplets, touching (including self-infection caused by contaminated hands), and short-distance transmission of respiratory aerosols of different sizes. Currently, SARS-CoV-2 is mainly spread via droplets. At first, this virus was transferred from bats to humans; it falls into a specific category of bat viruses. Different coronaviruses persist on surfaces for various lengths of time.

As 2019 ended, news arrived of an epidemic of pneumonia, with a few cases in a seafood wholesale market in Wuhan, China. Initially, a few cases were detected around December 8, and a cluster was revealed on approximately December 31,2019 , when the WHO office in China was given the information. The market was shut down on January 1, 2020, and the Chinese authority announced the viral threat. All active and suspected cases were tested. At that time, $\sim 300$ cases were positive and 4 people had died. Initially, few reports verified human-to-human transmission, and reports of super-spreading patients included 15 healthcare workers and viral spread to different Chinese cities. Various other countries also confirmed human-to-human transmission. After China, SARS-CoV-2 spread to Europe, across Asia, and throughout the rest of the world. On January 31, 2020, first case of COVID-19 was confirmed in Kerala, India, where a student tested positive as she returned from Wuhan, China. ${ }^{3,4}$ Presently, SARS-CoV-2 is still spreading throughout the world and has affected nearly 132,758 persons globally in 167

Author for correspondence: Karan Veer, E-mail: veerk@nitj.ac.in

Cite this article: Sharma P and Veer K. (2020). Action and problems related to the COVID-19 outbreak in India. Infection Control \& Hospital Epidemiology, 41: 1478-1479, https://doi.org/10.1017/ice.2020.186 countries. Throughout the world, the death rate is extremely high (Fig. 1, as of March 20, 2020).

COVID-19 has been declared a national disaster by the Indian government. ${ }^{6,7}$ The scientists at the Indian Council of Medical Research (ICMR) are continually obtaining global information related to the pandemic. They suggest the use of retroviral drugs. The ICMR is providing free and reliable testing and diagnosis to all individuals with symptoms of COVID-19. The government is trying to expand laboratory testing using Ministry of Health and Family Welfare (MOHFW) and non-ICMR laboratories in many facilities and organizations, such as the Council of Scientific \& Industrial Research (CSIR), the Department of Biotechnology (DBT), Defence Research, and the Development Organization (DRDO), and government medical colleges. Thus far, 15 laboratories in India are testing for SARS-CoV-2, and 19 will soon be added. ${ }^{8}$ The agencies in India conducting COVID-19 testing include the National Institute of Virology (NIV) in Pune, the Indian Council of Medical Research (ICMR) in Hyderabad, and the National Center for Disease Control (NCDC) in Delhi. All of these agencies work under the NIV. A fund named the COVID Fund for South Asian Association for Regional Cooperation (SAARC) Countries has been started by SAARC countries to fight COVID-19. In addition, the Indian government has appealed to its citizens to follow social distancing procedures, which is the most effective way to stop the community transmission of SARS-CoV-2.

The current COVID-19 situation has affected the whole world and has had a dramatic impact on India. In India, the death rate is comparatively good, but the recovery rate of infected persons is not, which is leading to a difficult situation in India. Infections are increasing day by day in India, even though community transmission began only recently. The Indian government has taken a few necessary steps to control the situation, such as making masks and sanitizer available and providing free testing and diagnosis. Public awareness and programs of "do's and don'ts" for COVID-19 are run at public places. Environmental conditions may also support controlling SARS-CoV-2; across Asia spring temperatures are increasing, which may decrease viral spread somewhat. Early prediction methods and a specific vaccine are not yet available, although government has been able to control the pandemic thus far. The World Health Organization (WHO) helps developing countries by providing funding, medical kits for testing, and proper guidance 